\title{
The Inbreeding Effective Population Number in Dioecious Populations
}

\author{
Thomas Nagylaki \\ Department of Ecology and Evolution, The University of Chicago, Chicago, Illinois 60637
}

Manuscript received August 5, 1994

Accepted for publication October 4, 1994

\begin{abstract}
The inbreeding effective population number in a dioecious population with discrete, nonoverlapping generations is investigated for both autosomal and $X$-linked loci. The recursion relations for the probabilities of genic identity and the effective population numbers are analyzed and compared in two cases: $(i)$ the offspring identified by sex in the calculation of the probability of common parentage and ( $i i)$ the offspring not so identified. Case $i$ gives the correct evolution of the probabilities of identity, but case $i i$ has been more widely studied and applied. A general symmetric framework that reduces the number of parameters is developed and used to examine a wide variety of models of panmixia and monogamy. Cases $i$ and $i$ agree in many, but not all, models.
\end{abstract}

$I^{\top}$ a population of $N$ monoecious individuals that mate wholly at random (including self-fertilization) and have a binomial distribution of the number of progeny, the probability of heterozygosity decreases by a fraction $1 /(2 N)$ per generation (WRIGHT 1931; MALÉCOT 1946, 1948; KIMURA 1963). Replacing $N$ by the inbreeding effective population number $N_{\text {e }}$ (we suppress the usual superscript or subscript $i$ ) extends, for long times, this fundamental property of an ideal population to populations with selfing forbidden, separate sexes, arbitrary progeny distributions, overlapping generations, and other biological complications (CROW and DenNiston 1988; CABallero 1994; and refs. therein ).

CROW and DENNISTON (1988) defined $N_{e}$ in terms of the probability of recent common ancestry of uniting gametes ( $c f$. Kimura and CROW 1963; Crow and KIMURA 1970, pp. 345-352; EWENS 1979, pp. 104-112; 1982 ) . In many cases, at least for large populations, this is equivalent to the above definition; in some situations, however, it describes only the effect of the first generation of inbreeding and does not give the correct asymptotic rate of decay of the probability of heterozygosity (CABALlero and Hill 1992), which is perhaps of more evolutionary importance.

CROW and DENNISTON (1988) used their definition to evaluate $N_{e}$ for a monoecious population with or without distinguishing sperm from eggs. With selfing prohibited, this distinction did not affect their result, but with selfing permitted, it did. CABALLERo and HILL (1992) noted, however, that in a large population of constant size, the inbreeding effective number defined in terms of the asymptotic decay of heterozygosity is independent of this distinction. Their result can be extended to variable population number.

Address for correspondence: Department of Ecology and Evolution, The University of Chicago, 1101 East 57th St., Chicago, IL 60637.
CRow and Denniston (1988) also evaluated $N_{e}$ for an autosomal locus in a dioecious population in two cases: $(i)$ the offspring identified by sex in the calculation of the probability of common parentage and ( $i i)$ the offspring not so identified. Again, their formula in case $i$ describes only the first generation of inbreeding, but now even the effective numbers derived from the asymptotic rate of decay of heterozygosity generally differ in the two cases (CABAllero and Hill 1992; CABALLERO 1995). The same discrepancy exists for $X$-linked loci (CABallero 1995). Even in those unusual situations in which offspring cannot be identified by sex, however, the correct asymptotic rate of decay is that in case $i$. Therefore, it is important to compare the analyses and results in the two cases. This paper is devoted to that task.

Although identifying offspring by sex can affect $N_{e}$ (CABALLERO 1995), the following argument shows that, even with unequal numbers of males and females and an arbitrary progeny distribution, in some models it does not do so.

Recall first that the variance effective population number $N_{e}^{(v)}$ is defined so as to preserve in complex biological situations the multinomial form of the variances and covariances of the allelic frequencies in an ideal population (CROW and DENNISTON 1988; NAGYLAKI 1992, pp. 261-267; CABALLERO 1994; and refs. therein). Since inbreeding coefficients and gene-frequency variances describe the same evolutionary process from different points of view, the two kinds of effective number should be equal, at least under simple conditions (CROW and Kimura 1970, p. 345; Hill 1972, p. 288). This will be the case if $N_{e}^{(v)}$ yields the asymptotic decay rate of the expected heterozygosity. In a monoecious population, if $N_{e}^{(v)}$ is large and constant, we can prove this result by replacing $N$ by $N_{e}^{(v)}$ in a derivation of KIMURA (1955, p. 431; see also Crow and 
KimURA 1970, pp. 330-331 ). In a dioecious population, if the standard diffusion approximation holds (ETHIER and NAGYLAKI 1980), the desired equality follows from replacing $N$ by $N_{e}^{(v)}$ in a proof in SVIREZHEV and PASSEKOV (1990, pp. 291-292). If the standard diffusion approximation does not hold, then $N_{\varepsilon}^{(v)}$ may not be constant ( $c f$. GILLESPIE 1975; EWENS 1979, p. 108).

Next, we note that, for both autosomal and $X$-linked loci, $N_{e}$ in case ii (MALÉCOT 1951; POLlak 1977; NAGYLAKI 1981, 1992, pp. 246-247) agrees asymptotically with the constant $N_{e}^{(v)}$ in the contingency-table and monogamy models of MORAN and WATTERSON (1959) and the general-progeny-distribution models of ETHIER and NAGYLAKI (1980). Since $N_{e}^{(v)}$ arises naturally in the analyses of MORAN and WATTERSON ( 1959 ) and ETHIER and NAGYLAKI (1980), we conclude (and shall prove explicitly below) that, in these models, identifying offspring by sex does not affect $N_{e}$.

In the next section, we shall write recursion relations for the probabilities of identity and derive from them expressions for the inbreeding effective population numbers for both autosomal and $X$-linked loci in a form that facilitates comparison of cases $i$ and $i i$. In the following section, we shall develop a general framework for the comparison and use it to examine a wide variety of stochastic models for panmixia in finite dioecious populations. Then we shall modify this framework so that it applies to monogamy, and we shall investigate the monogamous analogues of these panmictic models. We shall summarize and discuss our results in the final section.

Some authors focus on the implications of the result of a given experiment and treat the gametic output of the individuals as constants ( see, e.g., KIMURA and Crow 1963; Crow and KimURA 1970, pp. 345-365; Hill 1972, 1979; CROW and DENNISTON 1988; CABALLERO and HILL 1992; CABALlero 1995). However, in order to properly apply particular gametic distributions, such as the binomial or Poisson, and to investigate stochastic models, we must treat these gametic numbers as the random variables they actually are (see, e.g., POLLAK 1977, 1980, 1990; EWENS 1979, pp. 104-112; NAGYLAKI 1981, 1992, pp. 243-247, 261-267). Consult EwENs (1979, p. 111) for a lucid discussion of this point.

Generations are discrete and nonoverlapping; the population of $N_{1}$ males and $N_{2}$ females mates at random; mutation and selection are absent. We focus attention on a single multiallelic locus. The total population number is $N=N_{1}+N_{2}$.

\section{THE INBREEDING EFFECTIVE POPULATION NUMBER}

In this section, we shall write recursion relations for the probabilities of identity and derive from them expressions for the inbreeding effective population number $N_{e}$ in two cases: $(i)$ the offspring identified by sex in the calculation of the probability of common parentage and ( $i i)$ the offspring not so identified. Hence we shall derive necessary and sufficient conditions for the identity of the recursion relations in the two cases. We shall treat both autosomal and $X$-linked loci.

Autosomal loci: We shall analyze cases $i$ and $i$ in this order and then compare them.

Offspring identified by sex: Throughout this paper, the subscripts $q, r$, and $s$ will denote sex, 1 for males and 2 for females. We shall always use $q$ for parents and $r$ and $s$ for offspring. Let $P_{q, r s}$ represent the (constant) probability that two homologous genes from parents of sex $q$, one chosen at random from an offspring of sex $r$ and one from a different offspring of sex $s(\geq r)$, are derived from the same parent.

We designate by $f(t)$ the probability that an individual chosen at random in generation $t(=0,1,2, \ldots)$ is homozygous. We do not distinguish the probabilities of homozygosity in males and females because they are equalized by a single generation of random mating. Let $g_{r s}(t)$ signify the probability that two homologous genes in generation $t$, one chosen at random from an individual of sex $r$ and one from a different individual of sex $s(\geq r)$, are the same allele. At least in principle, these probabilities of identity in state are measurable in natural populations.

Our variables satisfy the recursion relations $(r, s=$ 1,$2 ; r \leq s$ )

$$
\begin{aligned}
f^{\prime}= & g_{12}, \\
g_{r s}^{\prime}= & \frac{1}{4}\left[P_{1, r s \frac{1}{2}}(1+f)+\left(1-P_{1, r s}\right) g_{11}\right] \\
& +\frac{1}{4}\left[P_{2, r s \frac{1}{2}}(1+f)+\left(1-P_{2, r s}\right) g_{22}\right]+\frac{1}{2} g_{12},
\end{aligned}
$$

where the prime indicates the next generation. Equation (1a) follows directly from the definitions of $f$ and $g_{12}$. To obtain ( $1 \mathrm{~b}$ ), note first that two genes chosen at random from distinct offspring are both paternal, both maternal, and one paternal and one maternal with respective probabilities $\frac{1}{4}, \frac{1}{4}$, and $\frac{1}{2}$, and then multiply these factors by the corresponding conditional probabilities of allelic identity of the two genes.

The probabilities of identity by descent satisfy not only (1), but also the particular initial conditions $f(0)$ $=g_{r s}(0)=0$ for every $r$ and $s$.

In accordance with our introductory discussion, we seek the maximal eigenvalue, $\lambda$, of $A$, the $4 \times 4$ matrix of coefficients in (1). We approximate $\lambda$ in the case of most evolutionary interest, large populations: $N_{1}, N_{2} \gg$ 1. Then the definition of $P_{q, r s}$ suggests that

$$
\epsilon \equiv \sum_{q, r, s} P_{4, r s} \ll 1
$$

indeed, we shall see that $\epsilon$ is generically of order $N_{1}^{-1}$ $+N_{2}^{-1}$. Therefore, we set 


$$
\lambda=1-\frac{1}{2 N_{e}}+O\left(\epsilon^{2}\right)
$$

as $\epsilon \rightarrow 0$, in which $O\left(\epsilon^{2}\right)$ denotes second-order terms in $\epsilon$.

We use perturbation theory to approximate $\lambda$ (FRANKLIN 1968, pp. 186-189; HORN and JOHNSON 1985, pp. 364-378). A glance at (1) informs us that

$$
A=A_{0}+A_{1} \text {, }
$$

where

$$
A_{0}=\frac{1}{4}\left(\begin{array}{cccc}
0 & 0 & 4 & 0 \\
0 & 1 & 2 & 1 \\
0 & 1 & 2 & 1 \\
0 & 1 & 2 & 1
\end{array}\right),
$$

The unperturbed matrix $A_{0}$ has the simple maximal eigenvalue 1 (which justifies the leading term in $\lambda$ ), with left and right eigenvectors

$$
U_{0}=\frac{1}{4}\left(\begin{array}{l}
0 \\
1 \\
2 \\
1
\end{array}\right), \quad V_{0}=\left(\begin{array}{l}
1 \\
1 \\
1 \\
1
\end{array}\right)
$$

these are normalized so that $U_{0}^{T} V_{0}=1$, where the superscript $T$ signifies transposition. We have

$$
-\frac{1}{2 N_{e}}=U_{0}^{T} A_{1} V_{0}
$$

which yields directly

$$
\begin{aligned}
N_{e}=16 /\left(P_{1,11}+2 P_{1,12}+P_{1,22}\right. & +P_{2,11} \\
& \left.+2 P_{2,12}+P_{2,22}\right) .
\end{aligned}
$$

It remains to evaluate $P_{q, r s}$ in terms of the means, variances, and covariances of the offspring distributions. Let $L_{q i, r}$ designate the (random) number of offspring of sex $r(=1,2)$ of the $i$ th $\left(i=1,2, \ldots, N_{q}\right)$ parent of sex $q(=1,2)$, and introduce

$$
\begin{aligned}
\mu_{q r}=\mathrm{E}\left(L_{q i, r}\right) & =N_{r} / N_{q}, \\
\sigma_{q r}^{2}=\operatorname{Var}\left(L_{q i, r}\right), \quad \sigma_{q, 12} & =\operatorname{Cov}\left(L_{q i, 1}, L_{q i, 2}\right) .
\end{aligned}
$$

Thus, $\mu_{q r}$ and $\sigma_{q r}^{2}$ denote the mean and variance of the number of offspring of sex $r$ of a parent of sex $q$, and $\sigma_{q, 12}$ represents the covariance of the number of sons and the number of daughters of a parent of sex $q$. Under our assumptions, these parameters do not depend on $i$.

Conditioned on a particular vector of offspring numbers $\mathbf{L}_{q, r}=\mathbf{l}_{q, r}$, the probability that two homologous genes from parents of sex $q$, chosen at random from distinct offspring of sex $r$, are derived from the same parent is

$$
\sum_{i=1}^{N_{q}}\left(\frac{l_{q i, r}}{N_{r}}\right)\left(\frac{l_{q i, r}-1}{N_{r}-1}\right)
$$

Therefore,

$$
\begin{aligned}
P_{q, r r} & =\sum_{\mathbf{1}_{q, r}} \mathrm{P}\left(\mathbf{L}_{q, r}=\mathbf{1}_{q, r}\right) \sum_{i=1}^{N_{q}} \frac{l_{q i, r}\left(l_{q i, r}-1\right)}{N_{r}\left(N_{r}-1\right)} \\
& =\mathrm{E}\left[\sum_{i=1}^{N_{q}} \frac{L_{q i, r}\left(L_{q i, r}-1\right)}{N_{r}\left(N_{r}-1\right)}\right] \\
& =\frac{N_{q}\left(\sigma_{q r}^{2}+\mu_{q r}^{2}-\mu_{q r}\right)}{N_{r}\left(N_{r}-1\right)} \\
& =\frac{1}{N_{r}-1}\left[\left(\frac{N_{q}}{N_{r}}\right) \sigma_{q r}^{2}+\frac{N_{r}}{N_{q}}-1\right] .
\end{aligned}
$$

Similarly,

$$
P_{q, 12}=\mathrm{E}\left[\sum_{i=1}^{N_{q}} \frac{L_{q i, 1} L_{q i, 2}}{N_{1} N_{2}}\right]=\left(\frac{N_{q}}{N_{1} N_{2}}\right) \sigma_{q, 12}+\frac{1}{N_{q}} .
$$

Substituting (9) into (7) yields

$$
\begin{aligned}
& \frac{1}{N_{e}} \approx \frac{1}{16}\left\{\frac { 1 } { N _ { 1 } } \left[2+\sigma_{11}^{2}+\left(\frac{N_{1}}{N_{2}}\right)^{2} \sigma_{12}^{2}\right.\right. \\
& \left.+2\left(\frac{N_{1}}{N_{2}}\right) \sigma_{1,12}\right] \\
& +\frac{1}{N_{2}}\left[2+\sigma_{22}^{2}+\left(\frac{N_{2}}{N_{1}}\right)^{2} \sigma_{21}^{2}\right. \\
& \left.\left.+2\left(\frac{N_{2}}{N_{1}}\right) \sigma_{2,12}\right]\right\} \text {. }
\end{aligned}
$$

Here and below, approximations always hold for $N_{1}, N_{2}$ $\gg 1$.

Equation (10) agrees with HiLL's (1972, 1979) formula for the variance effective population number. Inserting (9) into (1) gives the recursion relations of CABALlero and Hill (1992), who derived (10) by a different method (A. CABAllero, personal communication ).

Let $\sigma_{q}^{2}$ signify the variance of the total number of offspring,

$$
L_{q i}=L_{q i, 1}+L_{q i, 2}
$$

of a parent of sex $q$ :

$$
\sigma_{q}^{2}=\operatorname{Var}\left(L_{q i}\right)=\sigma_{q 1}^{2}+\sigma_{q 2}^{2}+2 \sigma_{q, 12} .
$$

If males and females are equally numerous $\left(N_{1}=N_{2}\right.$ $=\frac{1}{2} N$ ), then (10) reduces to (CABALLERo and HiLL 1992) 


$$
N_{e} \approx 8 N /\left(4+\sigma_{1}^{2}+\sigma_{2}^{2}\right) .
$$

If $\sigma_{q, 12}=0$ and $L_{q i, r}$ is binomially distributed with parameter $1 / N_{q}$ and index $N_{r}$ for every $q, r$, and $s$, then (9) yields

$$
P_{q, r s}=1 / N_{q}
$$

for every $q, r$, and $s$. Substituting (13) into (7) gives the classical result (WRIGHT 1931; MALÉCOT 1946, 1948; KIMURA 1963)

$$
N_{e}=4 N_{1} N_{2} / N
$$

Offspring not identified by sex: If the probabilities $P_{q, r s}$ are assumed to depend only on the parental sex $q$, then they may be replaced in (1) by $P_{q}$. From (7) we immediately obtain the inbreeding effective population number (the asterisk distinguishes case $i i$ )

$$
N_{e}^{*}=4 /\left(P_{1}+P_{2}\right),
$$

where (NAGYLAKI 1981)

$$
P_{q}=\frac{1}{N-1}\left[\left(\frac{N_{q}}{N}\right) \sigma_{q}^{2}+\frac{N}{N_{q}}-1\right]
$$

$\sigma_{q}^{2}$ is given by (11). Substituting (16) into (15) leads to

$$
\frac{1}{N_{e}^{*}}=\frac{1}{4(N-1)}\left(\frac{N_{1} \sigma_{1}^{2}+N_{2} \sigma_{2}^{2}}{N}+\frac{N_{1}}{N_{2}}+\frac{N_{2}}{N_{1}}\right) .
$$

This formula was derived asymptotically for $N \gg 1$ by MALÉCOT (1951) and exactly by POLLAK (1977), who showed also that it is equivalent to a result of KIMURA and CRow (1963); see also NAGYLAKI (1992, pp. 246247). Equation (17) agrees asymptotically with the variance effective numbers in the models of MORAN and WATTERSON (1959), FELDMAN (1966), and ETHIER and NAGYLAKI (1980).

If $N_{1}=N_{2} \gg 1$, we see at once that (17) reduces to $(12)$.

If $L_{q i}$ is binomial with parameter $1 / N_{q}$ and index $N$, as is consistent with the more detailed binomial hypothesis below (12), then from (16) we deduce

$$
P_{q}=1 / N_{q} \text {. }
$$

Inserting (18) into (15) again yields (14)

Comparison: The above treatment of cases $i$ and $i i$ proves that the recursion relations agree if and only if

$$
P_{q, r s}=P_{q}
$$

for every $q, r$, and $s$, where $P_{q, r}$ and $P_{q}$ are given by (9) and (16), respectively. Thus, the simpler case $i i$ gives the correct evolution of the probabilities of identity if and only if (19) holds.

From (7) and (15) we infer directly that the inbreeding effective population numbers in the two cases will be equal if

$$
\frac{1}{4} P_{q, 11}+\frac{1}{2} P_{q, 12}+\frac{1}{4} P_{q, 22}=P_{q}
$$

for $q=1,2$. Thus, ( 20 ) suffices for equality of the asymptotic rates of decay of the expected heterozygosity in the two cases. Of course, the condition (19) is stronger than (20).

In general, neither (19) nor (20) holds. Here and below, such assertions of failure apply even asymptotically as $N_{1}, N_{2} \rightarrow \infty$.

If $N_{1}=N_{2}$, then, although (19) fails generically, (20) holds asymptotically; as we have seen, $N_{e}$ is given by (12).

In the binomial case, (13) and (18) establish (19), and (14) specifies $N_{\rho}$.

$X$-linked loci: To investigate $X$-linked loci, we follow the above approach and indicate only the required modifications.

Offspring identified by sex: Instead of (1), now we have the recursion relations

$$
\begin{aligned}
f^{\prime}= & g_{12} \\
g_{11}^{\prime}= & P_{2,11} \frac{1}{2}(1+f)+\left(1-P_{2,11}\right) g_{22}, \\
g_{12}^{\prime}= & \frac{1}{2}\left[P_{2,12 \frac{1}{2}}(1+f)+\left(1-P_{2,12}\right) g_{22}\right]+\frac{1}{2} g_{12}, \\
g_{22}^{\prime}= & \frac{1}{4}\left[P_{1,22}+\left(1-P_{1,22}\right) g_{11}\right] \\
& +\frac{1}{4}\left[P_{2,22} \frac{1}{2}(1+f)+\left(1-P_{2,22}\right) g_{22}\right]+\frac{1}{2} g_{12},
\end{aligned}
$$

where $f$ now refers only to females. From (21) we can again read off the matrices $A_{0}$ and $A_{1}$; from $A_{0}$ we can easily find the normalized eigenvectors $U_{0}$ and $V_{0}$. Then (6) leads to

$$
N_{e}=9 /\left(2 P_{1,22}+P_{2,11}+2 P_{2,12}+P_{2,22}\right) .
$$

Substituting (9) into (22), we obtain

$$
\begin{aligned}
& \frac{1}{N_{e}} \approx \frac{1}{9}\left\{\frac{1}{N_{1}}\left[1+2\left(\frac{N_{1}}{N_{2}}\right)^{2} \sigma_{12}^{2}\right]\right. \\
& \left.+\frac{1}{N_{2}}\left[1+\sigma_{22}^{2}+\left(\frac{N_{2}}{N_{1}}\right)^{2} \sigma_{21}^{2}+2\left(\frac{N_{2}}{N_{1}}\right) \sigma_{2,12}\right]\right\} .
\end{aligned}
$$

Inserting (9) into (21) produces the recursion relations of CABAllero (1995), who derived (23) from them by a different method. POLLAK $(1980,1990)$ had obtained (23) earlier as a variance effective population number.

If $N_{1}=N_{2}$, then (23) simplifies to

$$
N_{e} \approx \frac{9 N}{4+4 \sigma_{12}^{2}+2 \sigma_{2}^{2}} .
$$

In the binomial case (13), from (22) we get the classical result (WRIGHT 1933; MALÉCOT 1951; KIMURA 1963) 


$$
N_{e}=\frac{9 N_{1} N_{2}}{4 N_{1}+2 N_{2}} .
$$

Offspring not identified by sex: If the probabilities $P_{q, s}$ in (21) are assumed to be independent of the offspring sexes $r$ and $s$, then they may be replaced by $P_{q}$. Substituting this into (22) yields the effective number (NAGYLAKI 1981)

$$
N_{e}^{*}=\frac{9}{2 P_{1}+4 P_{2}} .
$$

Inserting (16) into (26), we deduce (NAGYLAKI 1981)

$$
\frac{1}{N_{e}^{*}}=\frac{2}{9(N-1)}\left(\frac{N_{1} \sigma_{1}^{2}+2 N_{2} \sigma_{2}^{2}}{N}+\frac{2 N_{1}}{N_{2}}+\frac{N_{2}}{N_{1}}\right) \text {, }
$$

which agrees asymptotically with the variance effective population numbers in the models of MORAN and WATTERSON (1959) and ETHIER and NAGYLAKI (1980).

If $N_{1}=N_{2}$, then (27) simplifies to

$$
N_{e}^{*}=\frac{9(N-1)}{6+\sigma_{1}^{2}+2 \sigma_{2}^{2}} ;
$$

in contrast to the autosomal case, this generally differs from (24).

In the binomial case (18), we recover (25).

Comparison: A quick inspection of (21) demonstrates that the recursion relations in cases $i$ and $i i$ agree if and only if

$$
P_{1,22}=P_{1}, \quad P_{2, r s}=P_{2}
$$

for every $r$ and $s$. From (22) and (26) we infer immediately that $N_{e}^{*}=N_{e}$ if

$$
P_{1,22}=P_{1}, \quad \frac{1}{4} P_{2,11}+\frac{1}{2} P_{2,12}+\frac{1}{4} P_{2,22}=P_{2} .
$$

In the symmetric models analyzed in the next two sections, the conditions (29) and (30) hold only if the stronger condition (19) does, which we have established above only in the binomial case.

\section{MODELS OF PANMIXIA}

In the preceding section, we saw that case $i$, in which the offspring are identified by sex in the calculation of the probability of common parentage, and case $i i$, in which they are not so identified, are equivalent if the progeny distribution is binomial. Here, we shall investigate in far more generality and depth the conditions under which the two cases are equivalent.

The recursion relations ( 1 ) and the effective number (7) have six parameters: the four variances $\sigma_{q r}^{2}$ and the two covariances $\sigma_{q, 12}$. In the first subsection, we shall present a formulation in terms of exchangeable matings that reduces these to three. Then we shall apply this framework to some models of MORAN and WATTERSON
(1959) and ETHIER and NAGYLAKI (1980) and to a culling model.

Since we have simplified our problem to settling the validity of the condition (19), we can now treat autosomal and $X$-linked loci simultaneously.

General formulation: We assume that all possible matings occur. Then panmixia and the absence of mutation and selection imply that the progeny distribution of the different matings is exchangeable.

Some modification of the notation of the previous section will ease our analysis. Henceforth, we shall consistently denote males by $i$ and $k\left(=1,2, \ldots, N_{1}\right)$ and females by $j$ and $l\left(=1,2, \ldots, N_{2}\right)$. Let $K_{r, i j}$ designate the number of offspring of sex $r$ from the mating of male $i$ with female $j$. Then the total number of offspring from this mating is

$$
K_{i j}=K_{1, i j}+K_{2, i j} .
$$

Let $K_{r, i}$ and $K_{i}$, respectively, represent the number of offspring of sex $r$ and the total number of offspring of male $i$; the corresponding quantities for female $j$ are $K_{r, j}$ and $K_{\cdot j}$. In the notation of the previous section, we have

$$
\begin{array}{ll}
K_{r, i}=L_{1 i, r}, & K_{r \cdot j}=L_{2 j, r}, \\
K_{i,}=L_{1 i}, & K_{\cdot j}=L_{2 j} .
\end{array}
$$

As functions of our basic random variables $K_{r, i j}$, the variables in (32) are given by

$$
\begin{array}{ll}
K_{r, i}=\sum_{j=1}^{N_{2}} K_{r, i j}, & K_{r, j}=\sum_{i=1}^{N_{1}} K_{r, i j}, \\
K_{i,}=K_{1, i}+K_{2, i}, & K_{, j}=K_{\mathbf{1}, j}+K_{2, j} .
\end{array}
$$

Our variables satisfy the obvious constraint

$$
\sum_{i=1}^{N_{1}} \sum_{j=1}^{N_{2}} K_{r, i j}=N_{r} .
$$

Taking the expectation of (34) and appealing to exchangeability yields at once

$$
\mathrm{E}\left(K_{r, i j}\right)=\frac{N_{r}}{N_{1} N_{2}} ;
$$

(31) and (35a) give

$$
\mathrm{E}\left(K_{i j}\right)=\frac{N}{N_{1} N_{2}} .
$$

Let $\tau_{r}$ signify the variance of the number of offspring of sex $r$ from the mating of female $i$ with male $j$, and let $\gamma$ denote the covariance of the number of sons and the number of daughters from that mating:

$$
\tau_{r}=\operatorname{Var}\left(K_{r, i j}\right), \quad \gamma=\operatorname{Cov}\left(K_{1, i}, K_{2, i j}\right) .
$$

These are our three basic parameters. If $\sigma^{2}$ designates the variance of the total number of offspring from the 
mating of male $i$ with female $j$, then (31) and (36) inform us that

$$
\sigma^{2}=\operatorname{Var}\left(K_{i j}\right)=\tau_{1}+\tau_{2}+2 \gamma .
$$

We define the variances and covariances for individuals as in ( $8 \mathrm{~b})$ and $(1 \mathrm{~b})$, changing our notation according to (32):

$$
\begin{aligned}
\sigma_{1 r}^{2} & =\operatorname{Var}\left(K_{r, i}\right), \quad \sigma_{2 r}^{2}=\operatorname{Var}\left(K_{r, j}\right), \\
\sigma_{1,12} & =\operatorname{Cov}\left(K_{1, i \cdot}, K_{2, i \cdot}\right), \\
\sigma_{2,12} & =\operatorname{Cov}\left(K_{1, \cdot j}, K_{2, \cdot j}\right), \\
\sigma_{1}^{2} & =\operatorname{Var}\left(K_{i \cdot}\right), \quad \sigma_{2}^{2}=\operatorname{Var}\left(K_{\cdot j}\right) .
\end{aligned}
$$

From (33b) and (38) we recover (11b):

$$
\sigma_{q}^{2}=\sigma_{q 1}^{2}+\sigma_{q 2}^{2}+2 \sigma_{q, 12}
$$

In view of (37) and (39), it remains only to express the six parameters $\sigma_{q r}^{2}$ and $\sigma_{q, 12}$ as functions of the three parameters $\tau_{r}$ and $\gamma$.

Invoking exchangeability, from (34) we obtain

$$
\begin{aligned}
0=\operatorname{Var}\left(\sum_{i=1}^{N_{1}} \sum_{j=1}^{N_{2}} K_{r, i j}\right)=N_{1} N_{2} \operatorname{Var}\left(K_{r, i j}\right) \\
\quad+N_{1} N_{2}\left(N_{1} N_{2}-1\right) \operatorname{Cov}\left(K_{r, i j}, K_{r, k l}\right),
\end{aligned}
$$

in which $(i, j) \neq(k, l)$. We conclude

$$
\operatorname{Cov}\left(K_{r, i j}, K_{r, h l}\right)=-\frac{\tau_{r}}{N_{1} N_{2}-1} .
$$

Note that (40) would have to be modified for monogamy because, in that case, for $i \neq k$ and $j \neq l$,

$$
P\left(K_{r, i j}>0, K_{r, i l}>0\right)=0,
$$

whereas

$$
P\left(K_{r, i j}>0, K_{r, k l}>0\right)>0,
$$

which violates exchangeability.

From (36), (34), and exchangeability, we find

$$
\begin{aligned}
\gamma & =\operatorname{Cov}\left(K_{1, i j}, N_{2}-\sum_{(k, l) \neq(i, j)} K_{2, k l}\right) \\
& =-\left(N_{1} N_{2}-1\right) \operatorname{Cov}\left(K_{1, i j}, K_{2, k l}\right),
\end{aligned}
$$

where $(i, j) \neq(k, l)$. Therefore, we have

$$
\operatorname{Cov}\left(K_{1, i j}, K_{2, k l}\right)=-\frac{\gamma}{N_{1} N_{2}-1} .
$$

We are now ready to evaluate $\sigma_{q r}^{2}$ and $\sigma_{q, 12}$. To write our results concisely, we define $q^{*}$ as the sex opposite to $q$ :

$$
q^{*}=3-q= \begin{cases}2 & \text { if } q=1 \\ 1 & \text { if } q=2\end{cases}
$$

Appealing successively to (38a), (33a), exchangeability, (36), and (41), we find

$$
\begin{aligned}
\sigma_{1 r}^{2} & =\operatorname{Var}\left(\sum_{j=1}^{N_{2}} K_{r, i j}\right) \\
& =N_{2} \operatorname{Var}\left(K_{r, i j}\right)+N_{2}\left(N_{2}-1\right) \operatorname{Cov}\left(K_{r, i j}, K_{r, i l}\right) \\
& =B_{1} \tau_{r},
\end{aligned}
$$

where we define

$$
\begin{aligned}
B_{q} & =\frac{N_{q^{*}}^{2}\left(N_{q}-1\right)}{N_{1} N_{2}-1} \\
& \approx N_{q^{*} .}
\end{aligned}
$$

We compute $\sigma_{2 r}^{2}$ in the same manner and deduce

$$
\sigma_{q r}^{2}=B_{q} \tau_{r}
$$

For the covariances, (38b), (33a), exchangeability, (36), and (43) lead to

$$
\begin{aligned}
\sigma_{1,12}= & \operatorname{Cov}\left(\sum_{j=1}^{N_{2}} K_{1, i j}, \sum_{l=1}^{N_{2}} K_{2, i l}\right) \\
= & N_{2} \operatorname{Cov}\left(K_{1, i j}, K_{2, i j}\right) \\
& +N_{2}\left(N_{2}-1\right) \operatorname{Cov}\left(K_{1, i j}, K_{2, i l}\right) \\
= & B_{1} \gamma .
\end{aligned}
$$

Interchanging $N_{1}$ and $N_{2}$ yields $\sigma_{2,12}$, and we get

$$
\sigma_{q, 12}=B_{q} \gamma
$$

We insert (48), (50), and (37) into (39) to derive

$$
\sigma_{q}^{2}=B_{q} \sigma^{2}
$$

Substituting (48), (50), (51), and (46) into (9) and $(16)$, we find

$$
P_{q, q q}=\left(\frac{N_{q^{*}}^{2}}{N_{1} N_{2}-1}\right) \tau_{\varphi}
$$

$$
\begin{array}{r}
P_{q, q^{*} q^{*}}=\frac{1}{N_{q^{*}}-1}\left\{\left[\frac{N_{1} N_{2}\left(N_{q}-1\right)}{N_{1} N_{2}-1}\right] \tau_{q^{*}}\right. \\
\left.+\frac{N_{q^{*}}}{N_{q}}-1\right\},
\end{array}
$$

$$
P_{q, 12}=\left(\frac{N_{1} N_{2}-N_{q^{*}}}{N_{1} N_{2}-1}\right) \gamma+\frac{1}{N_{q}},
$$




$$
P_{q}=\frac{N_{1} N_{2}}{N(N-1)}\left[\left(\frac{N_{1} N_{2}-N_{q^{*}}}{N_{1} N_{2}-1}\right) \sigma^{2}+\frac{N}{N_{q}^{2}}\right]
$$

For $N_{1}, N_{2} \gg 1$, these are approximated by

$$
\begin{aligned}
& P_{q, q q} \approx\left(\frac{N_{q^{*}}}{N_{q}}\right) \tau_{q}, \\
& P_{q, q^{*} q^{*}} \approx\left(\frac{N_{q}}{N_{q^{*}}}\right) \tau_{q^{*}}+\frac{1}{N_{q}}-\frac{1}{N_{q^{*}}}, \\
& P_{q, 12} \approx \gamma+\frac{1}{N_{q}}, \\
& P_{q} \approx \frac{N_{1} N_{2}}{N^{2}}\left(\sigma^{2}+\frac{N}{N_{q}^{2}}\right) .
\end{aligned}
$$

A glance at (54) and (55) reveals that (19) and (29) do not hold generically, so cases $i$ and $i i$ still differ.

If $N_{1}=N_{2}$, then (46) yields

$$
B_{q} \equiv B=N_{1}^{2} /\left(N_{1}+1\right) \approx N_{1},
$$

and therefore (48), (50), and (51) inform us that the variances and covariances

$$
\sigma_{q r}^{2}=B \tau_{r}, \quad \sigma_{q, 12}=B \gamma, \quad \sigma_{q}^{2}=B \sigma^{2}
$$

are now independent of the parental sex $q$. This is not true, however, for the probabilities $P_{q, r s}$, so (19) and (29) still fail, though (20) is satisfied asymptotically, as we saw generally in the preceding section.

Inserting (54) into (7) and (22), in case $i$ we obtain

$$
\begin{aligned}
& \frac{1}{N_{e}} \approx \frac{1}{8}\left[\left(\frac{N_{2}}{N_{1}}\right) \tau_{1}+\left(\frac{N_{1}}{N_{2}}\right) \tau_{2}+2 \gamma+\frac{N}{N_{1} N_{2}}\right], \\
& \frac{1}{N_{e}} \approx \frac{1}{9}\left[\left(\frac{N_{2}}{N_{1}}\right) \tau_{1}+3\left(\frac{N_{1}}{N_{2}}\right) \tau_{2}+2 \gamma+\frac{N}{N_{1} N_{2}}\right]
\end{aligned}
$$

for autosomal and $X$-linked loci, respectively. In case $i i$, substituting (55) into (15) and (26) gives

$$
\begin{aligned}
& \frac{1}{N_{e}^{*}} \approx \frac{1}{4 N}\left[2\left(\frac{N_{1} N_{2}}{N}\right) \sigma^{2}+\frac{N_{1}}{N_{2}}+\frac{N_{2}}{N_{1}}\right], \\
& \frac{1}{N_{e}^{*}} \approx \frac{2}{9 N}\left[3\left(\frac{N_{1} N_{2}}{N}\right) \sigma^{2}+\frac{2 N_{1}}{N_{2}}+\frac{N_{2}}{N_{1}}\right]
\end{aligned}
$$

for autosomal and $X$-linked loci, respectively.

If $\gamma=0$ and $K_{r, i j}$ is binomially distributed with parameter $1 /\left(N_{1} N_{2}\right)$ and index $N_{r}$, then (52) and (53) yield (13) and (18), respectively, and hence we recover (14) and (25) .

Although we have assumed that all possible matings occur, scrutiny of our analysis shows that it applies also if a single mating produces all the offspring:

$$
\begin{aligned}
& \mathrm{P}\left(K_{r, i j}=N_{r}, r=1,2\right)=\frac{1}{N_{1} N_{2}}, \\
& \mathrm{P}\left(K_{r, i j}=0, r=1,2\right)=1-\frac{1}{N_{1} N_{2}}
\end{aligned}
$$

for every $i$ and $j$. Calculating $\tau_{r}, \gamma$, and $\sigma^{2}$ from (36) and (37) and substituting the results into (52) and (53) informs us that $P_{q, r s}=P_{q}=1$, as is obvious. Unsurprisingly, this reduces (1) and (21) to the recursion relations for sib mating.

A model of MORAN and W ATTERSON: Let

$$
\mathbf{K}=\left(K_{11}, K_{12}, \ldots, K_{1 N_{2}}, K_{21}, \ldots, K_{N_{1}, N_{2}}\right)
$$

denote the vector of total offspring numbers. Conditioned on $\mathbf{K}$, the first two moments of $K_{r, i j}$ are determined as in a contingency table (MORAN and WATTERSON 1959, p. 10):

$$
\mathrm{E}\left(K_{r, i j} \mid \mathbf{K}\right)=\left(\frac{N_{r}}{N}\right) K_{i j},
$$

$$
\operatorname{Var}\left(K_{r, i j} \mid \mathbf{K}\right)=\left[\frac{N_{1} N_{2}}{N^{2}(N-1)}\right] K_{i j}\left(N-K_{i j}\right) .
$$

From (31) and (59b) we get immediately

$$
\begin{aligned}
\operatorname{Cov}\left(K_{1, i j}, K_{2, i j} \mid \mathbf{K}\right) & \\
= & -\left[\frac{N_{1} N_{2}}{N^{2}(N-1)}\right] K_{i j}\left(N-K_{i j}\right) .
\end{aligned}
$$

We now express $\tau_{r}$ and $\gamma$ in terms of the single parameter $\sigma^{2}$. Using (36), (59b), (59a), (35b), and (37), we find

$$
\begin{aligned}
\tau_{r} & =\mathrm{E}\left[\operatorname{Var}\left(K_{r, i j} \mid \mathbf{K}\right)\right]+\operatorname{Var}\left[\mathrm{E}\left(K_{r, i j} \mid \mathbf{K}\right)\right] \\
& =\frac{1}{N-1}\left\{\left[\frac{N_{r}\left(N_{r}-1\right)}{N}\right] \sigma^{2}+1-\frac{1}{N_{1} N_{2}}\right\} \\
& \approx \frac{1}{N}\left[\left(\frac{N_{r}^{2}}{N}\right) \sigma^{2}+1\right], \\
\gamma & =\operatorname{E}\left[\operatorname{Cov}\left(K_{1, i j}, K_{2, i j} \mid \mathbf{K}\right)\right] \\
& =\frac{1}{N-1}\left[\left(\frac{N_{1} N_{2}}{N}\right) \sigma^{2}-1+\frac{1}{N_{1} N_{2}}\right] \\
& \approx \frac{1}{N}\left[\left(\frac{N_{1} N_{2}}{N}\right) \sigma^{2}-1\right] .
\end{aligned}
$$

Substituting (61) and (64) into (52) and comparing the simplified results with (53) establishes (19) exactly. Therefore, cases $i$ and $i i$ are identical. 
Inserting (62) and (65) into (56) yields

$$
\begin{aligned}
& \frac{1}{N_{p}} \approx \frac{1}{4 N}\left[2\left(\frac{N_{1} N_{2}}{N}\right) \sigma^{2}+\frac{N_{1}}{N_{2}}+\frac{N_{2}}{N_{1}}\right], \\
& \frac{1}{N_{e}} \approx \frac{2}{9 N}\left[3\left(\frac{N_{1} N_{2}}{N}\right) \sigma^{2}+\frac{2 N_{1}}{N_{2}}+\frac{N_{2}}{N_{1}}\right]
\end{aligned}
$$

for autosomal and $X$-linked loci, respectively.

If $K_{i j}$ is binomial with parameter $1 /\left(N_{1} N_{2}\right)$ and index $N$, then (53) leads to (18), and hence to (14) and (25).

A model of ETHIER and NagylakI: To formulate the dioecious general-progeny-distribution model of ETHIER and NAGYLAKI (1980), assume first that all the matings produce offspring independently, with the same progeny distribution, which has positive probabilities of producing 0 and 1 offspring. In each mating, conditioned on the total number of progeny of that mating, the distribution of the number of male (or female) progeny is binomial. Finally, condition to choose only those transitions in which the total number of progeny of sex $r$ is $N_{\text {, }}$.

In the APPENDIX, we shall prove that this scheme leads precisely to (61) and (64), so all the results in the preceding subsection are valid here.

Culling: Here, we formulate and analyze a model in the spirit of the diallelic, haploid culling scheme of GILLESPIE ( 1975 ). This model is more complicated and difficult, but probably more realistic, than the models in the preceding two subsections.

Our assumptions are the same as in the ETHIER-NAGYLAKI model described above, except that, instead of conditioning on $N_{r}$ offspring of sex $r$, we sample $N_{r}$ adults of sex $r$ from the juvenile offspring population. Sampling may be with or, more realistically, without replacement.

Generically, both (19) and (20) fail. In the APPENDIX, we shall derive the following explicit results for a Poisson (pre-culling) offspring distribution, in which case $\gamma=0$.

For sampling with replacement, let $\mu_{r}$ denote the mean of the total number of juvenile progeny of sex $r$; then

$$
\tau_{r} \approx \frac{1}{N_{r^{*}}}\left(1-\frac{1}{N_{1} N_{2}}\right)\left(1+\frac{N_{r}-1}{\mu_{r}}\right)
$$

if $\mu_{r} \gg 1$. Thus, (19) and (20) still fail unless $\mu_{r} \gg N_{r}$ for $r=1$ and 2, which yields the binomial case (13), (14), and (25), as expected. See Equation (A42) for the exact expression for $\tau_{r}$.

For sampling without replacement,

$$
\tau_{r}=\frac{1}{N_{r^{*}}}\left(1-\frac{1}{N_{1} N_{2}}\right),
$$

exactly as in the binomial case. Much more generally, at the end of the APPENDIX we shall prove that $\mathbf{K}_{r}=$
( $\left.K_{r, 11}, K_{r, 12}, \ldots, K_{r, N_{1} N_{2}}\right)$ is multinomially distributed. It is interesting to note that, with the appropriate choice of parameters, the diffusion coefficient in GiLLESPIE's (1975) model reduces to the binomial case for sampling without replacement, but not for sampling with replacement.

\section{MODELS OF MONOGAMY}

Here, we modify the general formulation and specific models of panmixia in the preceding section to explore monogamy. We assume that $N_{1}=N_{2}=\frac{1}{2} N$. Then every individual mates exactly once, pairing at random, and this greatly simplifies both formulation and analysis.

General formulation: We number the $N_{1}$ exchangeable matings according to the male involved $(i=1$, $\left.\ldots, N_{1}\right)$. Let $K_{r, i}$ designate the number of offspring of sex $r$ from mating $i$. Then the total number of offspring from this mating is

$$
K_{i}=K_{1, i}+K_{2, i},
$$

and

$$
\sum_{i=1}^{N_{1}} K_{r, i}=N_{1}
$$

Taking the expectation of ( 70) and invoking exchangeability yields immediately

$$
\mathrm{E}\left(K_{r, i}\right)=1 \text {, }
$$

whence

$$
\mathrm{E}\left(K_{i}\right)=2 .
$$

Our basic parameters are again $\tau_{r}$, the variance of the number of offspring of sex $r$ from mating $i$, and $\gamma$, the covariance of the number of sons and the number of daughters from that mating. Now the variances and covariances defined in ( $8 \mathrm{~b})$ and (11b) must be independent of parental sex and are given simply by

$$
\begin{aligned}
& \sigma_{q r}^{2}=\tau_{r}=\operatorname{Var}\left(K_{r, i}\right), \\
& \sigma_{q, 12}=\gamma=\operatorname{Cov}\left(K_{1, i}, K_{2, i}\right), \\
& \sigma_{\eta}^{2}=\sigma^{2}=\operatorname{Var}\left(K_{i}\right)=\tau_{1}+\tau_{2}+2 \gamma .
\end{aligned}
$$

Substituting (72) into (9) and (16) with $N_{1}=N_{2}$, we find

$$
\begin{aligned}
& P_{q, r r}=\frac{\tau_{r}}{N_{1}-1}, \\
& P_{q, 12}=\frac{\gamma+1}{N_{1}}, \\
& P_{q}=\frac{\sigma^{2}+2}{2(N-1)} .
\end{aligned}
$$

Generically, (19) fails, so cases $i$ and $i$ again differ. In accordance with our general result, if $N_{1}=N_{\mathrm{s}}$, then 
(19) still fails, but (20) holds asymptotically, whence $N_{e} \approx N_{e}^{*}$ for autosomal loci, but not for $X$-linked ones.

Inserting (72) into (12), (24), and (28), we get

$$
N_{e} \approx N_{e}^{*} \approx \frac{4 N}{2+\sigma^{2}}
$$

for autosomal loci (WRIGHT 1938, 1939) and

$$
\begin{aligned}
& N_{e} \approx \frac{9 N}{4+4 \tau_{2}+2 \sigma^{2}}, \\
& N_{e}^{*} \approx \frac{3 N}{2+\sigma^{2}}
\end{aligned}
$$

for $X$-linked ones (CABALlero 1995 ).

If $\gamma=0$ and $K_{r, i}$ is binomially distributed with parameter $1 / N_{1}$ and index $N_{1}$, then

$$
\tau_{r}=1-\frac{1}{N_{1}},
$$

and (73) and (74) give

$$
P_{q, r s}=P_{q}=1 / N_{1},
$$

in agreement (13) and (18). Consequently, (19) holds, and we find $N_{e}=N$ and $N_{e}=\frac{3}{4} N$ for autosomal and $X$-linked loci, respectively.

If each sibship consists of exactly one male and one female, then

$$
\tau_{r}=\gamma=\sigma^{2}=0,
$$

and hence $(73)$ and $(74)$ reduce to

$$
P_{q, r r}=0, \quad P_{q, 12}=\frac{1}{N_{1}}, \quad P_{q}=\frac{1}{N-1},
$$

which exemplifies the failure of (19). From (75) and (76) we obtain $N_{e} \approx N_{e}^{*} \approx 2 N$ for autosomal loci, and $N_{e} \approx \frac{9}{4} N$ and $N_{e}^{*} \approx \frac{3}{2} N$ for X-linked ones (CABALlero 1995 ).

A model of Moran and Watterson: Let

$$
\mathbf{K}=\left(K_{1}, K_{2}, \ldots, K_{N_{1}}\right)
$$

denote the vector of total offspring numbers. The conditional moments (59) still apply (MORAN and WATTERSON 1959, p. 13 ) :

$$
\begin{gathered}
\mathrm{E}\left(K_{r, i} \mid \mathbf{K}\right)=\frac{1}{2} K_{i}, \\
\operatorname{Var}\left(K_{r, i} \mid \mathbf{K}\right)=\frac{K_{i}\left(N-K_{i}\right)}{4(N-1)}, \\
\operatorname{Cov}\left(K_{1, i}, K_{2, i} \mid \mathbf{K}\right)=-\frac{K_{i}\left(N-K_{i}\right)}{4(N-1)} .
\end{gathered}
$$

We follow the analysis of the panmictic MORAN-WATTERSON model and express $\tau_{r}$ and $\gamma$ in terms of $\sigma^{2}$ :

$$
\begin{aligned}
\tau_{r} & =\left[\frac{N-2}{4(N-1)}\right]\left(\sigma^{2}+2\right) \\
& \approx \frac{1}{4}\left(\sigma^{2}+2\right), \\
\gamma & =\frac{4+N\left(\sigma^{2}-2\right)}{4(N-1)} \\
& \approx \frac{1}{4}\left(\sigma^{2}-2\right) .
\end{aligned}
$$

Substituting (83) and (85) into (73) demonstrates that

$$
P_{q, r s}=P_{q}=\frac{\sigma^{2}+2}{2(N-1)},
$$

which establishes (19).

The effective population numbers for autosomal and $X$-linked loci are given by (75) and ( $76 \mathrm{~b})$, respectively. If, in accordance with (47) and (51), we replace $\sigma^{2}$ in (66) by $\sigma_{q}^{2} / N_{1}$, we see that the effective population numbers for panmixia and monogamy are asymptotically equal, as shown by MORAN and WATTERSON (1959).

If $K_{i}$ is binomial with parameter $1 / N_{1}$ and index $N$, we again find $N_{e}=N$ and $N_{e}=\frac{3}{4} N$ for autosomal and $X$-linked loci, respectively.

A modification of the ETHIER-NAGYLAKI model: The description in the preceding section of the ETHIERNAGYLAKI model for panmixia applies unchanged to monogamy, and the mathematical analysis in the APPENDIX is easily adapted. We find that (83) and (85) hold exactly, so all the results in the preceding subsection are valid here.

Culling: Again, the description in the preceding section of the panmictic culling model applies unaltered, and the modification of the formulation and analysis in the APPENDIX is straightforward. For a Poisson (preculling) offspring distribution, we have $\gamma=0$ and the following results.

For sampling with replacement,

$$
\begin{aligned}
\tau_{r} & =\left(1-\frac{1}{N_{1}}\right)\left[1+\frac{\left(N_{1}-1\right) \psi\left(\mu_{r}\right)}{e^{\mu_{r}}-1}\right] \\
& \approx\left(1-\frac{1}{N_{1}}\right)\left(1+\frac{N_{1}-1}{\mu_{r}}\right)
\end{aligned}
$$

for $\mu_{r} \gg 1$, where $\mu_{r}$ denotes the mean of the total number of progeny of sex $r$, and the function $\psi$ is defined by Equations (A39) to (A41). As for panmixia, (19) and (20) fail unless $\mu_{1}, \mu_{2} \gg N_{1}$, which gives the binomial case (77) and (78).

For sampling without replacement, the binomial results (77) and (78) hold exactly.

\section{DISCUSSION}

Here, we summarize our main results and add some comments. 
We have presented straightforward, rigorous derivations of the inbreeding effective population numbers $N_{e}$ for autosomal and $X$-linked loci. The crucial parameters are the probabilities $P_{q, r s}$ of common parentage, which are given by (9) in terms of offspring variances $\sigma_{q r}^{2}$ and covariances $\sigma_{q, 12}$. If the possible dependence of $P_{q, r s}$ on the offspring sexes $r$ and $s$ is taken into account (case $i$ ), then $N_{e}$ is specified by (7) and (10) for autosomal loci and by (22) and (23) for X-linked ones. If this possible dependence is neglected (case $i i$ ), then the effective number (now called $N_{*}^{*}$ ) is determined by (15) and (17) for autosomal loci and by (26) and (27) for $X$-linked ones.

For autosomal loci, the probabilities of identity in cases $i$ and $i i$ satisfy the same recursion relations if and only if (19) holds, whereas (20) suffices for the equality $N_{e}=N_{e}^{*}$. For X-linked loci, the corresponding conditions are (29) and (30), respectively; in symmetric models such as the ones investigated here, however, (29) and (30) are equivalent to (19).

Cases $i$ and $i i$ are identical if the offspring distribution is binomial, and $N_{e}=N_{e}^{*}$ for an autosomal locus if the sexes are equally numerous $\left(N_{1}=N_{2}\right)$.

We formulated and investigated a model of panmixia in which all possible matings occur. In (48) and (50) we expressed the four offspring variances $\sigma_{q r}^{2}$ and the two offspring covariances $\sigma_{q, 12}$ for individuals in terms of the two variances $\tau_{r}$ and the covariance $\gamma$ for matings, whence (52) and (56) yield $P_{q, r s}$ and $N_{e}$ as functions of these three basic parameters. Cases $i$ and $i i$ (and $N_{e}$ and $N_{\varepsilon}^{*}$ ) still differ generically, but they agree in the models of MORAN and WATTERSON (1959) and ETHIER and NAGYLAKI (1980). They differ generically in culling models, except in the case of culling without replacement from a Poisson pre-culling offspring distribution, which leads to a binomial post-culling offspring distribution.

All the conclusions in the preceding paragraph hold for a model of monogamy with $N_{1}=N_{2}$. In (79) and (80), each sibship consists of exactly one male and one female, and this provides a simple example of the generic nonequivalence of cases $i$ and $i$.

Finally, suppose every allele mutates to new alleles at the same rate $u$. In NAGYLAKI (1981), a general matrix argument established the classical equilibrium result (MALÉCOT 1946, 1948, 1951; KIMURA and CROW 1964; CROW and KIMURA 1970, pp. 322-327)

$$
\hat{f} \approx \hat{g}_{r s} \approx 1 /\left(1+4 N_{e} u\right)
$$

for $u \ll 1$, for both autosomal and $X$-linked loci. That argument applies to both cases $i$ and $i i$, and therefore so does $(90)$.

I thank A. CABALleko for sending his stimulating paper to me before publication, and I am grateful to R. R. BAHADUR and S. L. ZABEIL for helpful discussions. This work was supported by National Science Foundation grant BSR-9006285.

\section{LITERATURE CITED}

Caballero, A., 1994 Developments in the prediction of effective population size. Heredity (in press).

Caballero, A., 1995 A note on the effective size for populations with separate sexes, with special reference to sex-linked genes. Genetics 139 (2) (in press).

Caballero, A., and W. G. Hill, 1992 A note on the inbreeding effective population size. Evolution 46: 1969-1972.

CROw, J. F., and C. DENNISTON, 1988 Inbreeding and variance effective population numbers. Evolution 42: $482-495$.

Crow, J. F., and M. KIMuRA, 1970 An Introduction to Population Genetics Theory. Harper \& Row, New York.

ETHIER, S. N., and T. NAGYLAKI, 1980 Diffusion approximations of Markov chains with two time scales and applications to population genetics. Adv. Appl. Probab. 12: 14-49.

EwENs, W.J., 1979 Mathematical Population Genetics. Springer-Verlag, Berlin.

EWENs, W. J., 1982 On the concept of effective population size. Theor. Popul. Biol. 21: 373-378.

Feldman, M. W., 1966 On the offspring number distribution in a genetic population. J. Appl. Prob. 3: 129-141.

FELLER, W., 1968 An Introduction to Probability Theory and Its Applications, Vol. I, Ed. 3. Wiley, New York.

Franki.IN, J. N., 1968 Matrix Theory. Prentice-Hall, Englewood Cliffs, NJ.

GaUTSCHI, W., and W. F. CAhill, 1964 Exponential integral and related functions, pp. 227-251 in Handbook of Mathematical Functions, edited by M. Abramowitz and I. A. STEgun. National Bureau of Standards, Washington, DC.

GILLESPIE, J. H., 1975 Natural selection for within-generation variance in offspring number. II. Discrete haploid models. Genetics 81: $403-413$.

HıLL, W. G., 1972 Effective size in populations with overlapping generations. Theor. Popul. Biol. 3: 278-289.

Hil., W. G, 1979 A note on effective population size with overlapping generations. Genetics 92: 317-322.

HorN, R. A., and C. R. JoHnson, 1985 Matrix Analysis. Cambridge University Press, Cambridge.

Johnson, N. L., and S. KoTz, 1969 Distributions in Statistics: Discrete Distributions. Houghton Mifflin, Boston.

KIMURA, M., 1955 Random genetic drift in multi-allelic locus. Evolution 9: 419-435.

KIMURA, M., 1963 A probability method for treating inbreeding systems, especially with linked genes. Biometrics 19: 1-17.

KimurA, M., and J. F. CROW, 1963 The measurement of effective population number. Evolution 17: 279-288.

KimurA, M., and J. F. Crow, 1964 The number of alleles that can be maintained in a finite population. Genetics 49: 725-738.

MALÉCOT, G., 1946 La consanguinité dans une population limitée. C. R. Acad. Sci. Paris 222: 841-843.

Malécot, G., 1948 Les mathêmatiques de l'hérédité, Masson, Paris.

MALÉCOT, G., 1951 Un traitment stochastique des problèmes linéaires (mutation, linkage, migration) en Génétique de Population. Ann. Univ. Lyon Sci. Sec. A 14: 79-117.

Moran, P. A. P., and G. A. WatTerson, 1959 The genetic effects of family structure in natural populations. Aust. J. Biol. Sci. 12: $1-15$.

NAGYLAKI, T., 1981 The inbreeding effective population number and the expected homozygosity for an $X$-linked locus. Genetics 97: $731-737$.

NAcil AKI, T., 1992 Introduction to Theoretical Population Genetics. Springer-Verlag, Berlin.

POLLAK, E., 1977 Effective population numbers and their interrelations, pp. 115-144 in Proceedings of the Washington State University Conference on Biomathematics and Biostatistics of May 1974. Department of Pure and Applied Mathematics, Washington State University, and Pi Mu Epsilon, Washington Alpha Chapter, Pullman, WA.

POLIAK, E, 1980 Effective population numbers and mean times to extinction in dioecious populations with overlapping generations. Math. Biosci. 52: 1-25.

Pollak, E., 1990 The effective population size of an age-structured population with a sex-linked locus. Math. Biosci. 101: 121-130. 
Svirezhev, Yu. M., and V. P. PASSEKov, 1990 Fundamentals of Mathematical Evolutionary Genetics. Kluwer, Dordrecht.

WRIGHT, S., 1931 Evolution in Mendelian populations. Genetics 16: 97-159.

Wright, S., 1933 Inbreeding and homozygosis. Proc. Natl. Acad. Sci. USA 19: 411-420.

WRIGHT, S., 1938 Size of population and breeding structure in relation to evolution. Science 87: 430-431.

Wright, S., 1939 Statistical Genetics in Relation to Evolution. Actual. Sci. Ind. 802: Exposés de biométrie et de statistique biologique. XIII, Edited by G. TeISSIER. Hermann, Paris.

Communicating editor: W. J. EwENs

\section{APPENDIX}

A model of ETHIER and NAGYLAKI: Here we prove (61) and (64) for the dioecious general-progeny-distribution model of ETHIER and NAGYLAKI (1980), which is an extension of FELDMAN's (1966) model.

Assume first that all matings produce offspring independently, with the same probability-generating function (p.g.f.) $F(\theta)$. In each mating, conditioned on the total number of progeny of that mating, each offspring independently has sex $r$ with probability $\pi_{r}$. Finally, condition to choose only those transitions in which the total number of progeny of sex $r$ is $N_{r}$. To ensure a positive probability for such reproductive events, we require that each mating produce 0 and 1 offspring with positive probabilities: $F(0)>0$ and $F^{\prime}(0)>0$. (In this APPENDIX, the prime indicates differentiation.) Then the p.g.f. of the offspring numbers $K_{r, i j}$ is ( $c f$. ETHIER and NAGYLAKI 1980, p. 44)

$$
\begin{aligned}
\&\left(\prod_{r=1}^{2} \prod_{i=1}^{N_{1}} \prod_{j=1}^{N_{2}} \rho_{r, i j}^{K_{r, i j}}\right) \\
=\frac{\left(\left(\theta_{1}^{N_{1}} \theta_{2}^{N_{2}}\right) \text { in } \prod_{i=1}^{N_{1}} \prod_{j=1}^{N_{2}} F\left(\theta_{1} \pi_{1} \rho_{1, i j}+\theta_{2} \pi_{2} \rho_{2, i j}\right)\right.}{\left({ }^{\circ}\left(\theta_{1}^{N_{1}} \theta_{2}^{N_{2}}\right) \text { in }\left[F\left(\theta_{1} \pi_{1}+\theta_{2} \pi_{2}\right)\right]^{N_{1} N_{2}}\right.},
\end{aligned}
$$

where $\theta\left(\theta_{1}^{N_{1}} \theta_{2}^{N_{2}}\right)$ denotes the coefficient of $\theta_{1}^{N_{1}} \theta_{2}^{N_{2}}$ in the function of $\theta_{1}$ and $\theta_{2}$ that follows it.

Observe that both the numerator and denominator contain $\pi_{r}$ only as a factor $\pi_{1}^{N_{1}} \pi_{2}^{N_{2}}$, so (A1) is actually independent of the underlying sex ratio.

To evaluate $\tau_{r}$ and $\gamma$ from (36), we require only the p.g.f. of the exchangeable random variables $K_{r, i j}$ for each $i$ and $j$, rather than the joint p.g.f. (A1). We set $\rho_{r, i j}=\xi_{r}$ for the mating under consideration and $\rho_{r, i j}=$ 1 for the others, and put

$$
\begin{gathered}
\theta=\theta_{1} \pi_{1}+\theta_{2} \pi_{2}, \quad G(\theta)=[F(\theta)]^{N_{1} N_{2}}, \\
c=\left(\left(\theta_{1}^{N_{1}} \theta_{2}^{N_{2}}\right) \text { in } G(\theta) .\right.
\end{gathered}
$$

Then (A1) informs us that the p.g.f.

$$
H\left(\xi_{1}, \xi_{2}\right)=\varepsilon_{1}\left(\xi_{1}^{K_{1, i j}} \xi_{2, i j}^{K_{2, i j}}\right)
$$

is given by

$$
\begin{aligned}
c H\left(\xi_{1}, \xi_{2}\right)= & \rho\left(\theta_{1}^{N_{1}} \theta_{2}^{N_{2}}\right) \text { in } \\
& {[F(\theta)]^{N_{1} N_{2}-1} F\left(\theta_{1} \pi_{1} \xi_{1}+\theta_{2} \pi_{2} \xi_{2}\right) . }
\end{aligned}
$$

According to (36), we wish to calculate

$\tau_{r}=\frac{\partial^{2} H}{\partial \xi_{r}^{2}}(1,1)-\left[\frac{\partial H}{\partial \xi_{r}}(1,1)\right]^{2}+\frac{\partial H}{\partial \xi_{r}}(1,1)$

$$
\gamma=\frac{\partial^{2} H}{\partial \xi_{1} \partial \xi_{2}}(1,1)-\frac{\partial H}{\partial \xi_{1}}(1,1) \frac{\partial H}{\partial \xi_{2}}(1,1) .
$$

Recalling (35a) and (44), we see from (A4) that

$$
\frac{\partial H}{\partial \xi_{r}}(1,1)=\xi_{i}\left(K_{r, i j}\right)=1 / N_{r^{*}},
$$

which can be verified by differentiating (A5).

From (A3) we obtain

$$
c=\left(\begin{array}{c}
N \\
N_{1}
\end{array}\right) \pi_{1}^{N_{1}} \pi_{2}^{N_{2}}\left[\left(\left(\theta^{N}\right) \text { in } G(\theta)\right] .\right.
$$

We define

$$
\begin{aligned}
\tilde{G}(\theta) & =\theta^{2} F^{\prime \prime}(\theta)[F(\theta)]^{N_{1} N_{2}-1}, \\
\beta & =\frac{{ }^{\rho}\left(\theta^{N}\right) \text { in } \tilde{G}(\theta)}{{ }^{\rho}\left(\theta^{N}\right) \text { in } G(\theta)} .
\end{aligned}
$$

Differentiating (A5) and inserting (A9) leads to

$$
\begin{aligned}
& c \frac{\partial^{2} H}{\partial \xi_{1}^{2}}(1,1) \\
& \quad=\left(\begin{array}{c}
N-2 \\
N_{1}-2
\end{array}\right) \pi_{1}^{N_{1}} \pi_{2}^{N_{2}}\left[\rho^{\prime}\left(\theta^{N}\right) \text { in } \tilde{G}(\theta)\right] .
\end{aligned}
$$

We substitute (A8) and (A10) into (A11) to derive

$$
\frac{\partial^{2} H}{\partial \xi_{r}^{2}}(1,1)=\left[\frac{N_{r}\left(N_{r}-1\right)}{N(N-1)}\right] \beta
$$

In the same manner, we find

$$
\frac{\partial^{2} H}{\partial \xi_{1} \partial \xi_{2}}(1,1)=\left[\frac{N_{1} N_{2}}{N(N-1)}\right] \beta \text {. }
$$

Inserting (A7), (A12), and (A13) into (A6) yields

$$
\begin{aligned}
& \tau_{r}=\left[\frac{N_{r}\left(N_{r}-1\right)}{N(N-1)}\right] \beta-\frac{1}{N_{r^{*}}^{2}}+\frac{1}{N_{r^{*}}}, \\
& \gamma=\left[\frac{N_{1} N_{2}}{N(N-1)}\right] \beta-\frac{1}{N_{1} N_{2}} .
\end{aligned}
$$

Substituting (A14) into (37), we deduce

$$
\sigma^{2}=\beta+\frac{N}{N_{1} N_{2}}\left(1-\frac{N}{N_{1} N_{2}}\right) .
$$


We solve (A15) for $\beta$ and insert the result into (A14) to establish (61) and (64).

Culling: Let $M_{r, i j}$ denote the number of juvenile offspring of sex $r$ from the mating of male $i$ with female $j$. The random vectors $\left(M_{1,11}, M_{2,11}\right)$, $\left(M_{1,12}, M_{2,12}\right), \ldots,\left(M_{1, N_{1} N_{2}}, M_{2, N_{1} N_{2}}\right)$ are independent and identically distributed. The total number of offspring from mating $i j$ is

$$
M_{i j}=M_{1, i j}+M_{2, i j} \text {. }
$$

The distribution of $M_{r, i}$, conditioned on $M_{i}$, is binomial with parameter $\pi_{r}$ and index $M_{i j}$. We designate the total number of offspring of sex $r$ by $M_{r}$ :

$$
M_{r}=\sum_{i=1}^{N_{1}} \sum_{j=1}^{N_{2}} M_{r, i j} .
$$

We define

$\mathbf{M}_{r}=\left(M_{r, 11}, M_{r, 12}, \ldots, M_{r, N_{1} N_{2}}\right), \quad \mathbf{M}^{*}=\left(\mathbf{M}_{1}, \mathbf{M}_{2}\right)$.

From the $M_{r}$ juvenile offspring of sex $r$, we sample $N_{r}$ adults, with or without replacement. Then $K_{r, i j}, K_{i j}, N_{r}$, $\mathbf{K}_{r}$, and $\mathbf{K}^{*}$ in adults correspond, respectively, to $M_{r, i}$, $M_{i j}, M_{r}, \mathbf{M}_{r}$, and $\mathbf{M}^{*}$ in offspring.

Thus, our model is

$$
\begin{aligned}
& \mathrm{P}\left(\mathbf{M}^{*}=\mathbf{m}^{*}\right) \\
& \quad=\prod_{i=1}^{N_{1}} \prod_{j=1}^{N_{2}}\left[\mathrm{P}\left(M_{i j}=m_{i j}\right)\left(\begin{array}{c}
m_{i j} \\
m_{1, i j}
\end{array}\right) \pi_{1}^{m_{1, i j}} \pi_{2}^{m_{2, i j}}\right], \\
& \mathrm{P}\left(\mathbf{K}^{*}=\mathbf{k}^{*}\right) \\
& \quad=\sum_{\mathbf{m}^{*}} \mathrm{P}\left(\mathbf{K}^{*}=\mathbf{k}^{*} \mid \mathbf{M}^{*}=\mathbf{m}^{*}\right) \mathrm{P}\left(\mathbf{M}^{*}=\mathbf{m}^{*}\right) .
\end{aligned}
$$

We shall not specialize $\mathrm{P}\left(M_{i j}=m_{i j}\right)$ to the Poisson offspring distribution until we must. To describe the culling, we must specify the conditional probability in (A19b).

For culling with replacement, we always condition on the event $M_{1}, M_{2}>0$ because otherwise the population becomes extinct, and we can make no observations. We shall indicate this conditioning only where necessary. Here we have the multinomial distribution

$$
\begin{aligned}
\mathrm{P}\left(\mathbf{K}^{*}\right. & \left.=\mathbf{k}^{*} \mid \mathbf{M} *=\mathbf{m}^{*}\right) \\
= & \prod_{r=1}^{2}\left[\left(\begin{array}{c}
N_{r} \\
k_{r, 11} \cdots k_{r, N_{2} N_{2}}
\end{array}\right) \prod_{i=1}^{N_{1}} \prod_{j=1}^{N_{2}}\left(\frac{m_{r, i j}}{m_{r}}\right)^{k_{r, i j}}\right] .
\end{aligned}
$$

For culling without replacement, we must condition on the event $M_{r} \geq N_{r}$ for $r=1$ and 2. Now we have the multivariate hypergeometric distribution (JOHNSON and KoTZ 1969, pp. 300-302)

$$
\begin{aligned}
\mathrm{P}\left(\mathbf{K}^{*}=\right. & \left.\mathbf{k}^{*} \mid \mathbf{M}^{*}=\mathbf{m}^{*}\right) \\
& =\prod_{r=1}^{2}\left\{\left[\prod_{i=1}^{N_{1}} \prod_{j=1}^{N_{2}}\left(\begin{array}{c}
m_{r, i j} \\
k_{r, i j}
\end{array}\right)\right] /\left(\begin{array}{c}
m_{r} \\
N_{r}
\end{array}\right)\right\} .
\end{aligned}
$$

We shall require the following means and variances. Taking conditional expectations in (A17) and invoking exchangeability, we obtain

$$
\mathrm{E}\left(R_{r, i j} \mid M_{1}, M_{2}\right)=\frac{1}{N_{1} N_{2}},
$$

where

$$
R_{r, i j}=M_{r, i j} / M_{r} .
$$

The distributions (A20) and (A21) have the same means (FELLER 1968, p. 233)

$$
\mathrm{E}\left(K_{r, i j} \mid \mathbf{M}^{*}\right)=N_{r} R_{r, i j} ;
$$

their variances are

$$
\operatorname{Var}\left(K_{r, i j} \mid \mathbf{M}^{*}\right)=N_{r} R_{r, i j}\left(1-R_{r, i j}\right)
$$

for (A20) and (FELLER 1968, p. 233)

$$
\operatorname{Var}\left(K_{r, i j} \mid \mathbf{M}^{*}\right)=\frac{N_{r} M_{r, i j}\left(M_{r}-M_{r, i j}\right)\left(M_{r}-N_{r}\right)}{M_{r}^{2}\left(M_{r}-1\right)}
$$

for (A21).

As always, we wish to evaluate $\tau_{r}$ and $\gamma$. From (A23) we get

$$
\begin{aligned}
\tau_{r} & =\mathrm{E}\left[\operatorname{Var}\left(K_{r, i j} \mid \mathbf{M}^{*}\right)\right]+\operatorname{Var}\left[\mathrm{E}\left(K_{r, i j} \mid \mathbf{M}^{*}\right)\right] \\
& =\mathrm{E}\left[\operatorname{Var}\left(K_{r, i j} \mid \mathbf{M}^{*}\right)\right]+N_{r}^{2} \operatorname{Var}\left(R_{r, i j}\right) .
\end{aligned}
$$

According to both (A20) and (A21), conditioned on $\mathbf{M}^{*}$, the random vectors $\mathbf{K}_{1}$ and $\mathbf{K}_{2}$ are mutually independent, as is obvious because the sexes are culled independently. Therefore,

$$
\operatorname{Cov}\left(K_{1, i j}, K_{2, i j} \mid \mathbf{M}^{*}\right)=0,
$$

and from (A23) we deduce

$$
\begin{aligned}
\gamma= & \mathrm{E}\left[\operatorname{Cov}\left(K_{1, i j}, K_{2, i j} \mid \mathbf{M}^{*}\right)\right] \\
& +\operatorname{Cov}\left[\mathrm{E}\left(K_{1, i j} \mid \mathbf{M}^{*}\right), \mathrm{E}\left(K_{2, i j} \mid \mathbf{M}^{*}\right)\right] \\
= & N_{\mathbf{1}} N_{2} \operatorname{Cov}\left(R_{1, i j}, R_{2, i j}\right) .
\end{aligned}
$$

We now analyze separately culling with and without replacement.

Culling with replacement: From (A26), (A24), and (A22) we find

$$
\tau_{r}=\frac{1}{N_{r^{*}}}\left(1-\frac{1}{N_{1} N_{2}}\right)+N_{r}\left(N_{r}-1\right) V_{r},
$$

where

$$
\begin{aligned}
V_{r}= & \operatorname{Var}\left(R_{r, i j} \mid M_{1}, M_{2}>0\right) \\
= & \mathrm{E}\left[\operatorname{Var}\left(R_{r, i j} \mid M_{1}, M_{2}\right) \mid M_{1}, M_{2}>0\right] \\
& +\operatorname{Var}\left[\mathrm{E}\left(R_{r, i j} \mid M_{1}, M_{2}\right) \mid M_{1}, M_{2}>0\right] \\
= & \mathrm{E}\left[M_{r}^{-2} \operatorname{Var}\left(M_{r, i j} \mid M_{1}, M_{2}\right) \mid M_{1}, M_{2}>0\right] .
\end{aligned}
$$


To proceed, we posit that the independent, identically distributed random variables $M_{i j}$ have a Poisson distribution with mean $\kappa$. Therefore, we have the joint p.g.f.

$$
\varepsilon\left(\prod_{r=1}^{2} \prod_{i=1}^{N_{1}} \prod_{j=1}^{N_{2}} \rho_{r, i j}^{M_{r, i j}}\right)=\prod_{i=1}^{N_{1}} \prod_{j=1}^{N_{2}} e^{\kappa\left(\pi_{1} \rho_{1, i j}+\pi_{2} \rho_{2, i j}-1\right)},
$$

whence (A17) gives

$$
\begin{aligned}
& \Leftrightarrow\left(\xi_{1}^{M_{1}} \xi_{2}^{M_{2}}\right)=e^{\kappa N_{1} N_{2}\left(\pi_{1} \xi_{1}+\pi_{2} \xi_{2}-1\right)}, \\
& \delta\left(\xi^{M_{r}}\right)=e^{\mu_{r}(\xi-1)},
\end{aligned}
$$

where $\mu_{r}=\kappa \pi_{r} N_{1} N_{2}$. Thus, the random vectors $\mathbf{M}_{1}$ and $\mathbf{M}_{2}$ are mutually independent, and the Poisson random variable $M_{r, i j}$ has mean $\kappa \pi_{r}$. The sums $M_{1}$ and $M_{2}$ are mutually independent Poisson random variables, and $M_{r}$ has mean $\mu_{r}$.

The above results yield the conditional multinomial distribution

$$
\begin{aligned}
P\left(\mathbf{M}_{r}=\mathbf{m}_{r} \mid M_{r}=\right. & \left.m_{r}\right)=P\left(\mathbf{M}_{r}=\mathbf{m}_{r}\right) / P\left(M_{r}=m_{r}\right) \\
= & \left(\begin{array}{c}
m_{r} \\
m_{r, 11} \cdots m_{r, N_{1} N_{2}}
\end{array}\right)\left(\frac{1}{N_{\mathrm{t}} N_{2}}\right)^{m_{r}}, \quad
\end{aligned}
$$

for which

$$
\operatorname{Var}\left(M_{r, i j} \mid M_{r}\right)=\frac{M_{r}}{N_{1} N_{2}}\left(1-\frac{1}{N_{1} N_{2}}\right) .
$$

Substituting (A35) into (A30) yields

$$
V_{r}=\frac{1}{N_{1} N_{2}}\left(1-\frac{1}{N_{1} N_{2}}\right) \&\left(M_{r}^{-1} \mid M_{r}>0\right) .
$$

From the Poisson distribution, we obtain

$$
\begin{gathered}
P\left(M_{r}>0\right)=1-e^{-\mu_{r},} \\
P\left(M_{r}>0\right) E\left(M_{r}^{-1} \mid M_{r}>0\right)=e^{-\mu_{r}} \psi\left(\mu_{r}\right),
\end{gathered}
$$

where

$$
\psi(\mu)=\sum_{n=1}^{\infty} \frac{\mu^{n}}{n(n !)}=\int_{0}^{\mu}\left(\frac{e^{x}-1}{x}\right) d x .
$$

This is essentially an exponential integral (GAUTSCHI and CAHILL 1964, pp. 228, 230):

$$
\psi(\mu)=\mathrm{Ei}(\mu)-\ln \mu-C,
$$

where $C$ represents EuLER's constant and

$$
\operatorname{Ei}(\mu)=\int_{-\infty}^{\mu}\left(\frac{e^{x}}{x}\right) d x
$$

in (A41), the principal value of the integral is understood. Inserting (A36), (A37), and (A38) into (A29) gives

$$
\tau_{r}=\frac{1}{N_{r^{*}}}\left(1-\frac{1}{N_{1} N_{2}}\right)\left[1+\frac{\left(N_{r}-1\right) \psi\left(\mu_{r}\right)}{e^{\mu_{r}}-1}\right] .
$$

As $\mu \rightarrow \infty$,

$$
\operatorname{Ei}(\mu) \sim \frac{e^{\mu}}{\mu}
$$

(GAUTSCHI and CAHILL 1964, pp. 228, 231), so (A40) and (A42) establish (67).

Since $\mathbf{M}_{1}$ and $\mathbf{M}_{2}$ are independent, from (A28) we conclude immediately that $\gamma=0$.

Culling without replacement: From (A26), (A25), and (A22) we derive

$$
\tau_{r}=\frac{N_{r}}{N_{1} N_{2}}\left(1-\frac{N_{r}}{N_{1} N_{2}}\right)+N_{r}\left(N_{r}-1\right) U_{r},
$$

where

$$
U_{r}=\varepsilon\left\{\varepsilon\left[\frac{M_{r, i j}\left(M_{r, i j}-1\right)}{M_{r}\left(M_{r}-1\right)} \mid M_{r}\right] \mid M_{s} \geq N_{s}, s=1,2\right\} .
$$

We now introduce the Poisson assumption (A31). Application of (A22) and (A35) reduces the inner expectation in (A45) to $\left(N_{1} N_{2}\right)^{-2}$, and substituting this into (A44) proves (68).

As for culling with replacement, $\gamma=0$.

In fact, (68) follows from the much more general result that, under the Poisson assumption (A31), the random vector $\mathbf{K}_{r}$ is multinomially distributed with equal probabilities $1 /\left(N_{1} N_{2}\right)$ and index $N_{r}$. To see this, first fix $m_{r} \geq N_{r}$ and condition $M_{r}=m_{r}$. According to (A31), the distribution of $\mathbf{M}_{r}$ is multinomial with equal probabilities $1 /\left(N_{1} N_{2}\right)$ and index $m_{r}$. Culling $N_{r}$ adults without replacement from these $m_{r}$ offspring yields $\mathbf{K}_{r}$. Therefore, conditioned on $M_{r}=m_{r}$, the distribution of $\mathbf{K}_{r}$ is multinomial with probabilities $1 /\left(N_{1} N_{2}\right)$ and in$\operatorname{dex} N_{r}$. Since this distribution is independent of $m_{r}$, it is identical to its average over $M_{r}$, which is the unconditional distribution of $\mathbf{K}$. 